\title{
Estimates of serial interval for COVID-19: A systematic review and meta- analysis
}

\author{
Balram Rai*, Anandi Shukla, Laxmi Kant Dwivedi \\ Department of Mathematical Demography and Statistics, International Institute for Population Sciences, Mumbai, 400088, India
}

\section{A R T I C L E I N F O}

\section{Keywords:}

COVID-19

Serial interval

Systematic review

Meta-analysis

Epidemiology

\begin{abstract}
A B S T R A C T
Background: On $11^{\text {th }}$ March 2020, the World Health Organization declared COVID-19 as Pandemic. The estimation of transmission dynamics in the initial days of the outbreak of any infectious disease is crucial to control its spread in a new area. The serial interval is one of the significant epidemiological measures that determine the spread of infectious disease. It is the time interval between the onset of symptoms in the primary and secondary case.

Objective: The present study aimed at the qualitative and quantitative synthesis of the currently available evidence for the serial interval of COVID-19.

Methodology: Data on serial intervals were extracted from 11 studies following a systematic review. A metaanalysis was performed to estimate the pooled estimate of the serial interval. The heterogeneity and bias in the included studies were tested by various statistical measures and tests, including $\mathrm{I}^{2}$ statistic, Cochran's $\mathrm{Q}$ test, Egger's test, and Beggs's test.

Result: The pooled estimate for the serial interval was $5.40(5.19,5.61)$ and $5.19(4.37,6.02)$ days by the fixed and random effects model, respectively. The heterogeneity between the studies was found to be $89.9 \%$ by $\mathrm{I}^{2}$ statistic. There is no potential bias introduced in the meta-analysis due to small study effects.

Conclusion: The present review provides sufficient evidence for the estimate of serial interval of COVID-19, which can help in understanding the epidemiology and transmission of the disease. The information on serial interval can be useful in developing various policies regarding contact tracing and monitoring community transmission of COVID-19.
\end{abstract}

\section{Introduction}

On $31^{\text {st }}$ December 2019, the outbreak of pneumonia cases of unknown etiology occurred in Wuhan and Hubei Province of China. ${ }^{1}$ As per the WHO's report, the outbreak was found to be associated with the seafood and the wet market in Wuhan. ${ }^{2}$ China reported the outbreak to the World Health Organization on 7th January 2020 stating that a new type of virus was identified as a coronavirus (2019-nCoV). ${ }^{3}$ The estimates from various reports of early stages show that the reproduction number of COVID-19 was found to greater than 1 (ranges between 2.24 and 3.58). ${ }^{4}$ It is a zoonotic coronavirus which is similar to SARS and MERS coronavirus. ${ }^{5}$ With the alarming levels of spread and severity, WHO declared the outbreak of COVID-19 as Pandemic on $11^{\text {th }}$ March $2020 .^{6}$

According to the World Health Organization, on 8th May 2020, there are 215 countries with approximately 3.7 million cases of COVID19 , with the death toll of around 0.26 million deaths. ${ }^{7}$ The transmissibility of COVID-19 from human to human is sufficient to support sustained transmission unless specific control measures are implemented. ${ }^{8}$ Hence, the estimation of transmission dynamics in the initial days of the outbreak of the disease becomes a thing of utmost importance. $^{9}$

The serial interval is one of the significant epidemiological measures that determine the spread of infectious disease. It is defined as the time for which the infector and infectee show the symptoms, i.e., the time interval between the onset of symptoms in the primary (infector) and secondary case (infectee). It is required to understand the turnover of case generation and transmissibility of the disease. ${ }^{10}$ It is often not easy to find links between the cases and hence, it becomes difficult to calculate the serial interval. The serial interval can also be a time dependent quantity, as its value can contract if the prevalence of disease increases during the course of pandemic. ${ }^{11}$ The serial interval consists of an incubation period (from infection to symptoms) and an infectious period (from exposure to infection). ${ }^{12}$ The serial interval is also referred

\footnotetext{
* Corresponding author. Department of Mathematical Demography and Statistics, International Institute for Population Sciences, Mumbai, 400088, India.

E-mail address: balramrai009@gmail.com (B. Rai).
} 


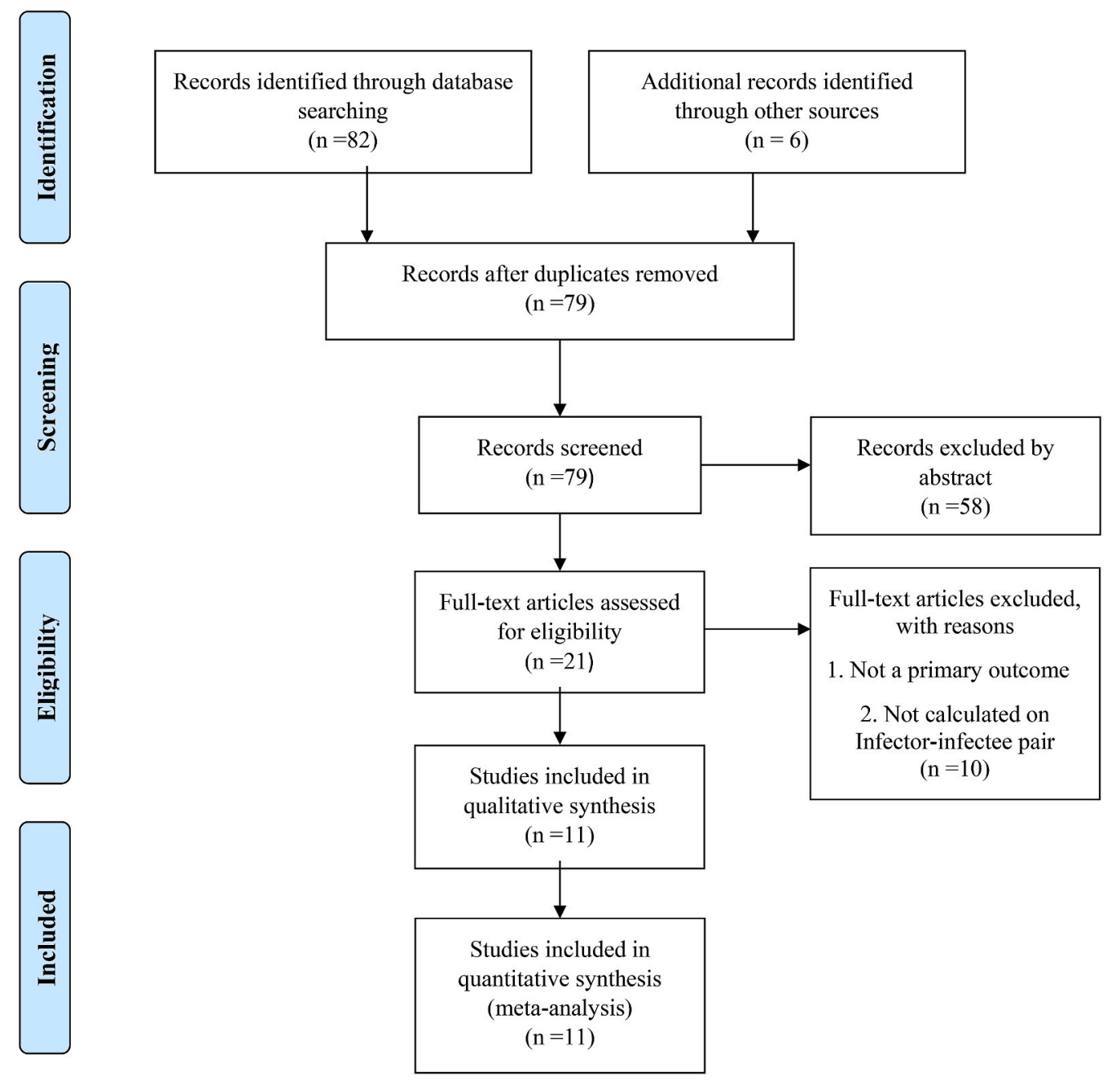

Fig. 1. Flow Chart for the selection of studies in systematic review and meta-analysis.

as generation time, which is defined as the average time taken for the secondary cases to be infected by a primary case. ${ }^{13}$ It is used in various epidemiological models such as SEIR, SIR, etc. which are used for the prediction of new cases and outbreaks in the community. The estimation of reproductive number can be sensitive to the choice of serial interval. ${ }^{14}$ The precision in the estimation of serial interval lead towards the accurate estimation of reproductive number, which is helpful in understanding the growth of any infectious disease. ${ }^{15}$ The serial interval can be used in disease surveillance. It is also useful in studies of predictive epidemiology and outbreak studies as it helps to identify the epidemiological link between the infector and infectee. ${ }^{16}$ The utility of this indicator can also be seen in studying the impact of various interventions and to inform authorities on how to control and mitigate outbreaks of any infectious disease. The present study focuses on the currently available evidence of the serial interval for COVID-19 and aims at qualitative and quantitative synthesis of the results obtained from the studies related to serial interval of COVID-19 pandemic.

\section{Methodology}

\subsection{Search databases and strategies}

The literature on the serial interval for COVID-19 was searched through popular research databases, including Google Scholar, Scopus, PubMed, and Science Direct, and google search. The search was accomplished by using the combination of keywords as "COVID 19 and serial interval", "2019-nCoV and serial interval", "Coronavirus and serial interval". We also performed a manual search by checking the reference list of the articles found in the search to avoid the omission of any eligible and important study. There was no restriction in the time period while searching the literature to possibly attain all the literature on the serial interval of COVID-19 to till date.

\subsection{Inclusion and exclusion criteria}

Inclusion criteria were as follows:

a) The study should be published in the English language.

b) The serial interval must be a primary outcome of the study.

c) The serial interval must be calculated based on the transmission chain of infections or symptoms of infector - infected pairs.

Exclusion criteria were as follows:

a) Duplicate studies.

b) The serial interval is taken as an input in the model from a different study.

\subsection{Data extraction from the studies}

All the articles were selected based on the inclusion and exclusion criteria. After selecting the final list of articles, the information regarding the name of the first author, study region, time period for data collection, adopted methodology in the study to estimate serial interval, 
estimates of the serial interval, and its $95 \%$ confidence interval were extracted from the study. The $95 \%$ confidence interval was calculated for the studies reporting mean serial interval with its standard deviation by using the following formula

$95 \% C I=\mu \pm 1.96\left(\frac{s}{\sqrt{n}}\right)$

where $\mu=$ mean serial interval, $s=$ standard deviation, and $\mathrm{n}=$ sample size of the study. A study has reported mean serial interval with its range, the standard deviation of that study was approximated to 'Range/4' by assuming the normal distribution of the serial intervals. ${ }^{17,18}$

\subsection{Statistical analyses}

All the meta-analyses were performed using Stata 14.1 software. The pooled effect size was calculated using fixed and random effects model. The heterogeneity between the studies was examined by Cochran's Q statistic and was quantified by Higgin's \& Thompson's I ${ }^{2}$ statistic and Tau-squared $\left(\tau^{2}\right)$. The bias introduced in the meta-analysis by small study effects was tested by Egger's and Begg's test for smallstudy effects. The Forest plot for the meta-analysis was plotted by using the random-effect model. A funnel plot at $95 \%$ confidence interval was also plotted to testify the publication bias in the meta-analysis.

\section{Results}

Altogether 88 studies were identified through initial retrieval from search databases. After implementing the inclusion and exclusion criteria, a total of 11 studies with 12 estimates of the serial interval were included in the meta-analysis of serial interval of COVID-19. Fig. 1describes the complete flowchart for selection of the studies in the qualitative and quantitative synthesis of the serial interval for COVID19 (Fig. 1). Since the dynamics of COVID-19 is still under exploration and researchers are exploring it worldwide, we included both published literature in peer-reviewed journals and available preprints. The available literature in the form of preprint cannot be ignored in view of current pandemic situation. However, we have not included any incomplete study or working paper in the qualitative or quantitative synthesis.

\subsection{Review of the included studies in the meta-analysis}

Table 1 describes the characteristics of the included studies in the meta-analysis of the serial interval of COVID-19. The mean serial
Table 2

Summary statistics for meta-analysis.

\begin{tabular}{ll}
\hline $\begin{array}{l}\text { Overall Effect Size } \\
\text { Fixed Effect Model } \\
\text { Random Effect Model }\end{array}$ & $5.40(5.19,5.61)$ \\
\hline $\begin{array}{l}\text { Test for Heterogeneity } \\
\mathrm{I}^{2} \text { Statistic }\end{array}$ & $5.19(4.37,6.02)$ \\
Tau squared $\left(\tau^{2}\right)$ & $89.9 \%$ \\
Cochran's Q & 1.4384 \\
\hline Small Study Effects & $109.17^{*}$ \\
Egger's test ${ }^{\mathrm{a}}$ & \\
Begg's test $^{\mathrm{a}}$ & $p=0.679$ \\
\hline$* \mathrm{p}<0.05$. & $p=0.631$ \\
${ }^{\mathrm{a}} \mathrm{H}_{0}:$ There are no small study effects. &
\end{tabular}

interval varies from $3.95(2.80,5.10)$ to $7.50(4.10,10.9)$ days in the included studies. Since the evidence of coronavirus was first found in China, we have included seven studies from China. Other than these, single study from each country Singapore, Hong Kong, Korea, and one with global data are also used in the review. The majority of the studies have used parametric distribution to compute the mean and standard deviation of the serial interval, including gamma and normal distribution. Four studies used the Bayesian approach to compute the serial interval from the transmission chain of infections. The period of data collection for computing serial interval was from the end of January 2020 till the start of March 2020 in the studies. The sample size of infector - infected pairs varies considerably from 6 to 689 in the included studies for meta-analysis [Table 1].

\subsection{Summary statistics for meta-analysis}

Table 2 describes the summary statistics after performing the metaanalysis for the included studies. The pooled estimate for the serial interval was $5.40(5.19,5.61)$ and $5.19(4.37,6.02)$ days by the fixed and random effects model, respectively. The heterogeneity between the studies was found to be $89.9 \%$ by I $^{2}$ statistic. The Cochran's Q test was statistically significant at $5 \%$ level of significance, suggesting the evidence for the heterogeneity between the included studies. The value for Tau squared $\left(\tau^{2}\right)$ was found to be 1.4834 . The Egger's test and Begg's test also suggested that there is no potential bias introduced in the meta-analysis due to small study effects [Table 2].

The forest plot (Fig. 2) provides the effect size of the individual studies with their weightage in the pooled estimate and a pooled estimate of the serial interval by random effect model, i.e., 5.19 (4.37,

Table 1

Characteristics of the included studies in the meta-analysis of serial interval (SI) of COVID-19.

\begin{tabular}{|c|c|c|c|c|c|c|c|}
\hline Authors & Study Area & Time Period & Methodology & Sample Size & SI & \multicolumn{2}{|c|}{$95 \% \mathrm{CI}$} \\
\hline Hiroshi Nishiura et al. ${ }^{19}$ & World & Up to $12^{\text {th }}$ February 2020 & Bayesian Approach with double interval censoring & 28 & 4.7 & 3.7 & 6.0 \\
\hline Zhanwei Du et al. ${ }^{20}$ & China & 21 st January $-8^{\text {th }}$ February 2020 & Fitting a normal distribution & 468 & 3.96 & 3.53 & 4.39 \\
\hline Qun Li et al. ${ }^{21}$ & Wuhan & Up to $22^{\text {nd }}$ January 2020 & Fitting a gamma distribution for laboratory-confirmed cases & 6 & 7.5 & 4.1 & 10.9 \\
\hline Moran $\mathrm{Ki}^{17}$ & Korea & Up to $20^{\text {th }}$ January 2020 & Calculating the mean of differences in time of symptoms & 7 & 6.3 & 4.1 & 8.5 \\
\hline Juanjuan Zhang et al. ${ }^{22}$ & China & $\begin{array}{l}\text { 19th January }-17^{\text {th }} \text { February } \\
2020\end{array}$ & Fitting a gamma distribution & 35 & 5.1 & 1.3 & 11.6 \\
\hline Shi Zhao et al. ${ }^{23}$ & Hong Kong & $\begin{array}{l}\text { 16th January }-15^{\text {th }} \text { February } \\
2020\end{array}$ & Fitting a gamma distribution & 21 & 4.4 & 2.9 & 6.7 \\
\hline Ganyani Tapiwa et al. ${ }^{24}$ & Singapore & $\begin{array}{l}21 \text { st January }-26^{\text {th }} \text { February } \\
2020\end{array}$ & Bayesian Framework & 27 & 5.2 & 3.6 & 6.8 \\
\hline Ganyani Tapiwa et al. ${ }^{24}$ & Tianjin (China) & $\begin{array}{l}\text { 14th January }-27^{\text {th }} \text { February } \\
2020\end{array}$ & Bayesian Framework & 57 & 3.9 & 2.8 & 5.1 \\
\hline Shujuan Ma et al. ${ }^{25}$ & Seven Countries & 29th February $-2^{\text {nd }}$ March 2020 & Fitting a normal distribution & 689 & 6.7 & 6.3 & 7.1 \\
\hline Qifang Bi et al. ${ }^{26}$ & China & $\begin{array}{l}\text { 14th January }-12^{\text {th }} \text { February } \\
2020\end{array}$ & Fitting a gamma distribution & 48 & 6.3 & 5.2 & 7.6 \\
\hline Choung You et al. ${ }^{27}$ & China & Up to 2nd February 2020 & Calculating the mean of differences in time of symptoms & 71 & 4.4 & 3.7 & 5.1 \\
\hline Menghui Li et al. ${ }^{28}$ & China & $\begin{array}{l}21 \text { st January }-29^{\text {th }} \text { February } \\
2020\end{array}$ & $\begin{array}{l}\text { Bayesian Approach with the doubly interval-censored } \\
\text { likelihood }\end{array}$ & 337 & 5.8 & 5.4 & 6.2 \\
\hline
\end{tabular}




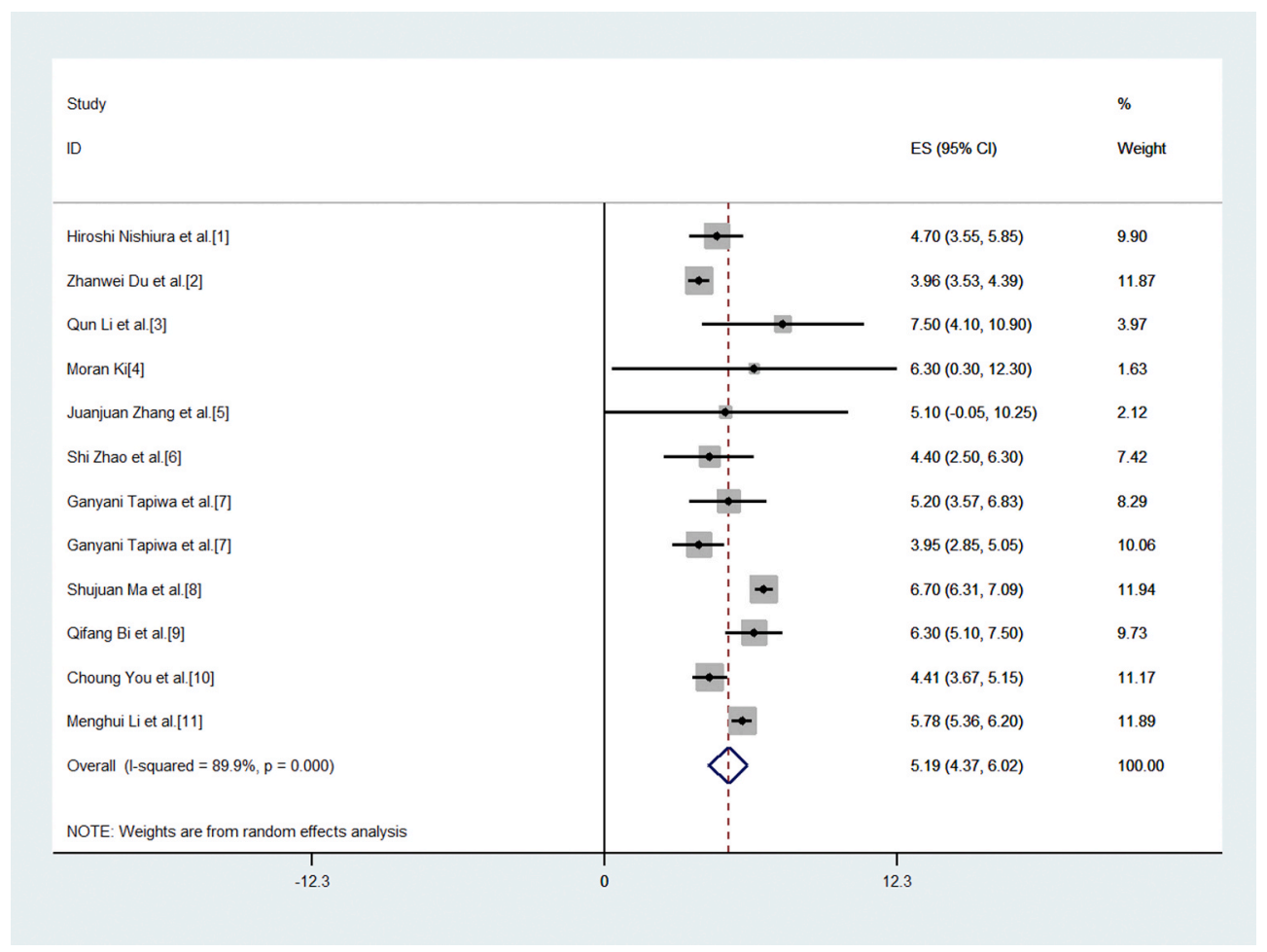

Fig. 2. Forest plot for the meta-analysis of serial interval for COVID-19.

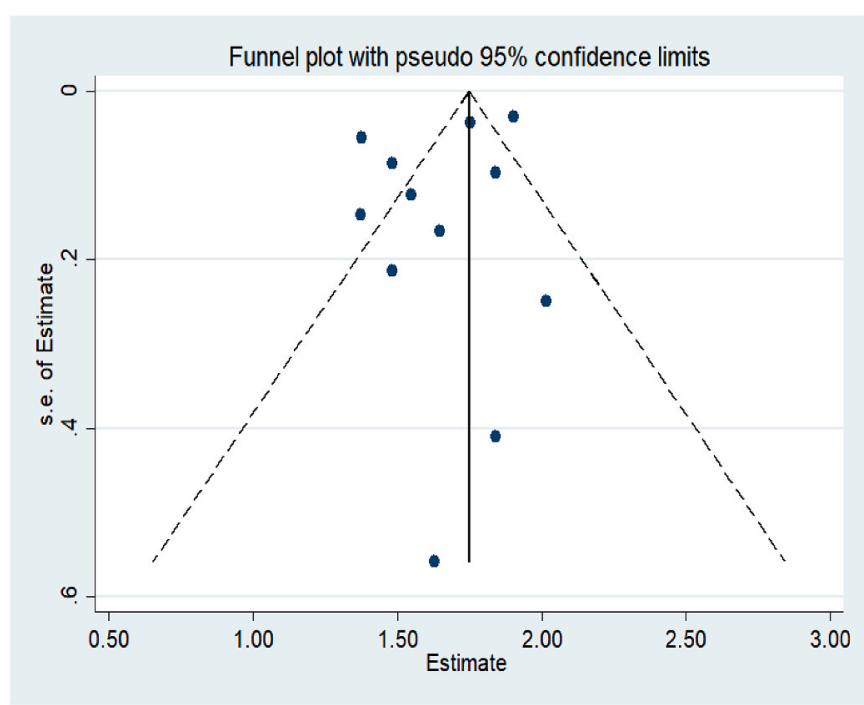

Fig. 3. Funnel Plot with a 95\% confidence interval for included studies in the meta-analysis.

6.02). The funnel plot (Fig. 3) with pseudo $95 \%$ confidence interval was plotted for the included studies in the meta-analysis, which suggests that there is no noticeable bias in the meta-analysis of studies. The little asymmetry in the funnel plot may be attributed to reporting bias and inadequate methodology adopted in the study. ${ }^{29}$

\section{Discussion}

The systematic review, in addition to meta-analysis, provides an opportunity to examine the existing literature and critically evaluate it with a statistical combination of results of comparable studies. ${ }^{30}$ The present meta-analysis provides sufficient evidence for the serial interval of COVID-19 through various studies, which can help in understanding the epidemiology and transmission of the disease. This review included 11 studies with 12 estimates of serial interval consisting data from total 1794 infector - infectee pairs of transmission, which used different methods to estimate the serial interval. The time period for the included studies varied from $14^{\text {th }}$ January 2020 to $2^{\text {nd }}$ March 2020. Initial studies provided a higher estimate of serial interval of around 6 to 7 , which significantly reduced to 4 to 5 in the later studies. The pooled estimate from random effect model is more appropriate due to the presence of considerable heterogeneity in the included studies for the meta- analysis. ${ }^{31}$ The pooled estimate was also consistent with some studies. ${ }^{19,22,24}$ The statistical tests also suggested the absence of any potential bias in the included studies for meta-analysis. Hence, the present meta-analysis provides a valid and reliable estimate of serial interval for COVID-19.

The pooled estimate of serial interval for COVID-19 from this meta analysis i.e. $5.19(4.37,6.02)$ days was lower than the serial interval of other similar infectious diseases such as, MERS [7.6 (2.5, 23.1) days] and SARS [ $8.4(\mathrm{SD}=3.8)$ days]. ${ }^{32,33}$ The serial interval of human influenza is $[3.6(2.9,4.3)$ days] which is lower than serial interval of COVID-19. ${ }^{34}$ The other infectious diseases such as smallpox (SI $=17.7$ days), measles (SI $=11.7$ days), rubella (SI $=18.3$ days) and mumps (SI $=18$ days) have higher serial interval. ${ }^{16}$ The estimation of serial interval is also important from policy perspectives, particularly for formulating the control strategies for reducing the growth of infection in the new area. The information on serial interval is useful for the contact tracing of infected cases and monitoring the community transmission of any infectious disease. The shorter serial interval of COVID-19 suggests that rapid contract tracing and case isolation is necessary to curtail the growth of infection in the next generation cases. The estimate of serial interval is also relevant for surveillance of infectious disease as it provides interval for reporting new cases infected by primary or index case. The information on serial interval along with the incubation period can be used as an aid to check the asymptomatic transmission of COVID-19. ${ }^{35}$

The differences in serial intervals may be attributable to several factors, including the nature of contacts and incidence of infection, 
which can vary among studies conducted at different times in different places. ${ }^{12,36}$ The precision of estimates of the serial interval can be improved further when detailed data on transmission, i.e., who-infectedwhom becomes available in the near future. ${ }^{16}$ However, the epidemiological investigation of COVID-19 is still in under investigation globally. In that case, this review can provide insights into the early estimation of the serial interval, which is an important characteristic of any infectious disease. The studies included in this analysis were mostly from China and its provinces; however, it would be better to include studies with a broad geographical scope to get a more comprehensive understanding of serial interval for COVID-19.

\section{Funding}

There was no funding for the study.

\section{Declaration of competing interest}

All the authors declare no conflict of interests.

\section{References}

1. Lu H, Stratton CW, Tang Y. Outbreak of pneumonia of unknown etiology in wuhan China: the mystery and the miracle. J Med Virol. 2020:25678.

2. World Health Organization. Available at: https://www.who.int/csr/don/12-january2020-novel-coronavirus-china/en/; 2020.

3. Corman VM, Landt O, Kaiser M, et al. Detection of 2019 novel coronavirus (2019nCoV) by real-time RT-PCR. Euro Surveill. 2020;25(3)

4. Zhao S, Lin $\mathrm{Q}$, Ran J, et al. Preliminary estimation of the basic reproduction number of novel coronavirus (2019-nCoV) in China, from 2019 to 2020: a data-driven analysis in the early phase of the outbreak. Int J Infect Dis:IJID: Off Publ Int Soc Infect Dis. 2020;92:214-217. https://doi.org/10.1016/j.ijid.2020.01.050.

5. Liu Y, Gayle AA, Wilder-Smith A, Rocklöv J. The reproductive number of COVID-19 is higher compared to SARS coronavirus. J Trav Med. March 2020;27(2):taaa021.

6. World Health Organization. Available at: https://www.who.int/docs/defaultsource/coronaviruse/transcripts/who-audio-emergencies-coronavirus-pressconference-full-and-final11 mar2020.pdf?sfvrsn $=$ cb432bb3 2; 2020

7. World Health Organization. Coronavirus disease 2019 (COVID-19). situation report. 2020:107 Available at: https://www.who.int/docs/default-source/coronaviruse/ situation-reports $/ 20200506$ covid-19-sitrep-107.pdf?sfvrsn =159c3dc 2 .

8. Natsuko Imai, Cori Anne, Dorigatti Ilaria, et al. Report 3: Transmissibility of 2019 nCoV. COVID-19 Response Team Imperial College London; 2020 Available at: https:// www.imperial.ac.uk/media/imperial-college/medicine/sph/ide/gida-fellowships/ Imperial-College-COVID19-transmissibility-25-01-2020.pdf.

9. Viboud C, Sun K, Gaffey R, et al. The RAPIDD ebola forecasting challenge: synthesis and lessons learnt. Epidemics. 2018;22:13-21.

10. Fine PEM. The interval between successive cases of an infectious disease. Am J Epidemiol. 2003;158(11):1039-1047.

11. Kenah E, Lipsitch M, Robins JM. Generation interval contraction and epidemic data analysis. Math Biosci. 2008;213(1):71-79.

12. Svensson $\AA$. A note on generation times in epidemic models. Math Biosci. 2007;208(1):300-311.

13. Anderson RM, Fraser C, Ghani AC, et al. Epidemiology, transmission dynamics and control of SARS: the 2002-2003 epidemic. Phil Trans Roy Soc Lond B Biol Sci. 2004;359(1447):1091-1105

14. Wallinga J, Lipsitch M. How generation intervals shape the relationship between growth rates and reproductive numbers. Proc Biol Sci. 2007;274(1609):599-604.

15. White LF, Wallinga J, Finelli L, et al. Estimation of the reproductive number and the serial interval in early phase of the 2009 influenza A/H1N1 pandemic in the USA. Influenza Other Respir Viruses. 2009;3(6):267-276.

16. Vink MA, Bootsma MCJ, Wallinga J. Serial intervals of respiratory infectious diseases: a systematic review and analysis. Am J Epidemiol. 2014:180(9):865-875.

17. Ki M. Epidemiologic characteristics of early cases with 2019 novel coronavirus (2019-nCoV) disease in Korea. Epidemiol Health. 2020;42.

18. Hozo SP, Djulbegovic B, Hozo I. Estimating the mean and variance from the median, range, and the size of a sample. BMC Med Res Methodol. 2005;5(1).

19. Nishiura H, Linton NM, Akhmetzhanov AR. Serial interval of novel coronavirus (COVID-19) infections. Int J Infect Dis. April 2020;93:284-286.

20. Du Z, Xu X, Wu Y, Wang L, Cowling BJ, Meyers LA. Serial interval of COVID-19 among publicly reported confirmed cases. Emerg Infect Dis. 2020;26(6):1341.

21. Li Q, Guan X, Wu P, Wang X, Zhou L, Tong Y, et al. Early transmission dynamics in Wuhan, China, of novel coronavirus-infected pneumonia. $N$ Engl J Med. 2020;382:1199-1207.

22. Zhang J, Litvinova M, Wang W, et al. Evolving epidemiology and transmission dynamics of coronavirus disease 2019 outside Hubei province, China: a descriptive and modelling study. Lancet Infect Dis. July 2020;20(7):793-802.

23. Zhao S, Gao D, Zhuang Z, Chong M, Cai Y, Ran J, et al. Estimating the serial interval of the novel coronavirus disease (COVID-19): a statistical analysis using the public data in Hong Kong from 16th January to 15th February, 2020. medRxiv. 2020. https://doi.org/10.1101/2020.02.21.20026559.

24. Ganyani T, Kremer C, Chen D, et al. Estimating the generation interval for coronavirus disease (COVID-19) based on symptom onset data, March 2020. Euro Surveill. 2020;25(17):2000257.

25. Ma S, Zhang J, Zeng M, Yun Q, Guo W, Zheng Y, et al. Epidemiological parameters of coronavirus disease 2019: a pooled analysis of publicly reported individual data of 1155 cases from seven countries. medRxiv. 2020. https://doi.org/10.1101/2020.03. 21.20040329.

26. Bi Q, Wu Y, Mei S, Ye C, Zou X, Zhang Z, et al. Epidemiology and transmission of COVID-19 in 391 cases and 1286 of their close contacts in Shenzhen, China: a retrospective cohort study. Lancet Infect Dis. August 2020;20(8):911-919.

27. You C, Deng Y, Hu W, Sun J, Lin Q, Zhou F, et al. Estimation of the time-varying reproduction number of COVID-19 outbreak in China. Int J Hyg Environ Health. 2020:113555.

28. Li M, Liu K, Song Y, Wang M, Wu J. Serial interval and generation interval for respectively the imported and local infectors estimated using reported contact-tracing data of COVID-19 in China. medRxiv. 2020. https://doi.org/10.1101/2020.04.15. 20065946.

29. Sterne JA, Sutton AJ, Ioannidis JP, Terrin N, Jones DR, Lau J, et al. Recommendations for examining and interpreting funnel plot asymmetry in metaanalyses of randomised controlled trials. Br Med J. 2011;343:d4002.

30. Fagard RH, Staessen JA, Thijs L. Advantages and disadvantages of the meta-analysis approach. J Hypertens. 1996;14(2):S9-S12 Suppl: Official journal of the International Society of Hypertension.

31. Borenstein M, Hedges L, Rothstein H. Meta-analysis: fixed effect vs. random effects Metaanalysis. com. Retrieved from https://www.metaanalysis.com/downloads/ Metaanalysis\%20fixed\%20effect\%20vs\%20random\%20effects\%20072607.pdf; 2007 Accessed on Jun 5, 2020.

32. Assiri A, McGeer A, Perl TM, et al. Hospital outbreak of Middle East respiratory syndrome coronavirus. N Engl J Med. 2013;369(5):407-416.

33. Lipsitch M, Cohen T, Cooper B, Robins JM, Ma S, James L, et al. Transmission dynamics and control of severe acute respiratory syndrome. Science. 2003;300(5627):1966-1970.

34. Cowling BJ, Fang VJ, Riley S, Peiris JM, Leung GM. Estimation of the serial interval of influenza. Epidemiology (Cambridge, Mass.). 2009;20(3):344.

35. Fraser C, Riley S, Anderson RM, Ferguson NM. Factors that make an infectious disease outbreak controllable. Proc Natl Acad Sci Unit States Am. 2004;101(16):6146-6151.

36. te Beest DE, Wallinga J, Donker T, van Boven M. Estimating the generation interval of influenza A (H1N1) in a range of social settings. Epidemiology. 2013:244-250. 